\title{
Matrix-Bracketing Blanks \& Peak Overlap Correction for Ti-V-Cr in Diverse Tourmalines
}

Jared Wesley Singer $^{1}$ and Marian Lupulescu ${ }^{2}$

${ }^{1}$ Troy, New York, United States, ${ }^{2}$ New York State Museum, Albany, New York, United States

This study gives a method for correction of the Ti-V-Cr peak overlaps and trace-level baselines for tourmalines by EPMA using a strategy of matrix-bracketing blanks. This case is a particular challenge for several reasons: 1) typical concentration levels are $\mathrm{Ti}>>\mathrm{V}>>\mathrm{Cr}$, 2) chromium suffers loss of precision from the cascading double interference correction, 3) common reference materials for interference calibration are not matrix matched (e.g. V2O3, Chromite), and 4) lack of high-purity tourmaline references which span wide range of compositions.

To circumvent the last of these issues, we solve the lack of ideal matrix-matched blanks using a population survey approach. LA-ICP-MS is used to rapidly identify candidate blanks from a compositionally diverse collection of natural tourmalines (mostly from New York State Museum and RPI collections). Of these a subset is identified based on particular characteristics such as homogeneity, having minimums and maximums of major elements (e.g. compositional bracketing), and critically, non-detectable levels $(<<$ $1 \mathrm{ppm}$ ) of a particular trace element. The blank population defines a trend line in the bivariate plot (see figure 1), where the slope is due to peak overlap and the intercept due to baseline subtraction. This scheme is used in conjunction with software-based interference correction algorithms, or can be used alone for post-hoc adjustment in the trace element limit. Donovan et al. (1993) pointed out a common mistake of ZAF miscalculation related to peak overlap calibration--exacerbated when reference materials were not matrix-matched. We find persistent bias of peak overlap corrections in modern software and recommend this empirical approach to double-check theoretical data processing.

Significant disagreement persists for the absolute inter-calibration of EPMA and LA-ICP-MS (commonly 0 to $30 \%$ bias is observed). For primary calibration of EPMA we still rely on high purity oxides (e.g. TiO2, V2O3, Chromite) calibrated at low beam current ( 10nA, but not 5nA due to an observed instability at the 5nA value). For LA-ICPMS we rely only on its fidelity to orders-of-magnitude (ppm > ppb, etc.). The ICPMS baseline is defined by the time-resolved gas-blank of the plasma, and common artifacts such as isobaric and molecular interference result in false positives. Care must be given to ensure that there is a reasonable ablation yield (nice tracks) and not overloading the plasma (suppressed ionization). The combination of techniques results in especially useful synergy when utilizing EPMA for accurate quantification and LA-ICPMS for checking the zero point of EPMA. We find that optimal precision for $\mathrm{Cr}$ is maintained by ensuring equally precise measurement of all $\mathrm{Ti}, \mathrm{V}$, and $\mathrm{Cr}$. 


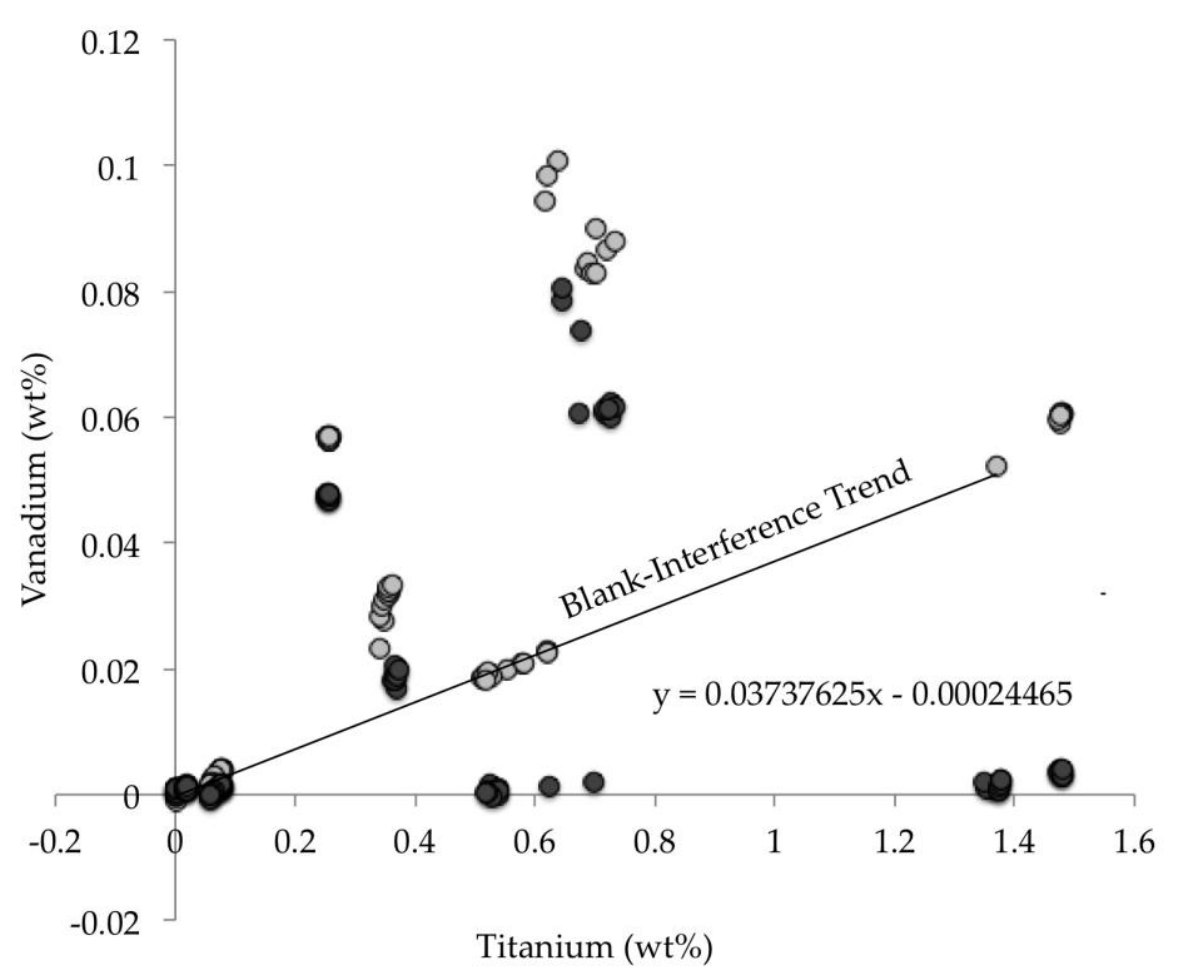

Figure 1. Bivariate plot of peak overlap. Light-grey symbols are for the original data biased by Ti-V peak overlap. The blank-interference trend line is indicated through samples which have zero vanadium. Dark symbols show transformation of the same data after peak overlap correction with matrix-bracketing blanks.

References

J. J. Donovan, D. A. Snyder and M. L. Rivers, "An Improved Interference Correction for Trace Element Analysis" Microbeam Analysis, 2: 23-28, 1993 